\title{
Association mapping of blood pressure levels in a longitudinal framework using binomial regression
}

\author{
Arunabha Majumdar, Indranil Mukhopadhyay, Saurabh Ghosh* \\ From Genetic Analysis Workshop 18 \\ Stevenson, WA, USA. 13-17 October 2012
}

\begin{abstract}
Heritable quantitative characters underline complex genetic traits. However, a single quantitative phenotype may not be a suitably good surrogate for a clinical end point trait.

It may be more optimal to use a multivariate phenotype vector correlated with the end point trait to carry out an association analysis. Existing methods, such as variance components and principal components, suffer from inherent limitations, such as lack of robustness or difficulty in biological interpretation of association findings. In an effort to circumvent these limitations, we propose a novel regression approach based on a conditional binomial model to detect association between a single-nucleotide polymorphism and a multivariate phenotype vector. We use our proposed method to analyze data on systolic and diastolic blood pressure levels provided in Genetic Analysis Workshop 18. We find that the bivariate analysis of the two phenotypes yields more promising results in terms of lower $p$-values compared to univariate analyses.
\end{abstract}

\section{Background}

Most clinical end point traits are governed by a set of quantitative precursors and a single precursor is unlikely to explain a significant amount of the variation in the end point trait. Thus, it may be a prudent strategy to analyze a multivariate vector of phenotypes for association mapping of a clinical end point trait. The major statistical challenge in the analyses of multivariate phenotypes lies in the modeling of the vector of phenotypes. Univariate analyses of the constituent phenotypes may lead to the statistical problem of multiple comparisons [1]. Likelihood-based methods, such as variance components [2,3], are susceptible to the choice of the joint probability distribution of the components of the vector. An alternative approach that circumvents the problem of modeling the multivariate phenotype is to obtain a reduced univariate phenotype using principal components [4]. However, association results based on principal components may be difficult to interpret biologically. We propose a novel binomial regression approach that models the likelihood of the number of copies of the minor allele at a single-nucleotide

\footnotetext{
* Correspondence: saurabh@isical.ac.in

Human Genetics Unit, Indian Statistical Institute, 203 B.T. Road, Kolkata
} 700108 , India

\section{Data description}

For our analyses, we use data on systolic blood pressure (SBP) levels and diastolic blood pressure (DBP) levels on 157 unrelated individuals along with their genotypes at all the available 456,752 variant sites distributed over 11 autosomal chromosomes in the genome-wide association studies data provided in GAW18. In addition to age, we use smoking status and medication indicator (both defined as binary variables) at each time point of examination as covariates, as these factors could be potential confounders in the association analyses. Both the SBP and the DBP levels are adjusted for these covariates for each time point and the tests for association are performed on the adjusted phenotypes. The adjustment and the association analyses are based on 139 individuals, as 
data on all the covariates are not available for the remaining individuals. The percentages of individuals with missing phenotype data at the different time points are $8 \%$, $35 \%, 35 \%$, and $75 \%$, respectively. On tabulating the percentages of individuals with phenotype data missing at multiple time points, we do not observe any pattern in the missing data. For our association analyses, we impute the missing data using a procedure outlined in the following section.

\section{Methods}

\section{Statistical methodology}

Imputation of missing phenotype values and covariate adjustment

The assumption of multivariate normality provides a computationally elegant framework for the expectation maximization algorithm [5] to estimate parameters when data are missing. Blood pressure levels have traditionally been believed to follow a lognormal distribution. While the Kolmogorov-Smirnov test did not show any significant departure from normality for the systolic and diastolic blood pressure levels at any of the time points, some of the $p$-values are very close to the threshold of 0.05 . We thus use a logarithmic transformation on each of the phenotypes to induce normality. Suppose the vector of log-transformed values of any of the two phenotypes at the 4 time points is represented as $X=\left(X_{1}, X_{2}\right.$, $X_{3}, X_{4}$ ). If $Y$ denotes the vector comprising those components of $X$ that are missing and $Z$ comprising the components that are available for an individual, $Y$ is estimated via an expectation maximization algorithm as the expectation of $\boldsymbol{Y}$ conditioned on $\boldsymbol{Z}$ and is given by $\boldsymbol{\mu}_{\mathrm{Y}}-\boldsymbol{\Sigma}_{\mathrm{YZ}} \boldsymbol{\Sigma}_{\mathrm{ZZ}}{ }^{-1}\left(\mathrm{Z}-\boldsymbol{\mu}_{\mathrm{Z}}\right)$, where, $\boldsymbol{\mu}_{\mathrm{Y}}$ and $\boldsymbol{\mu}_{\mathrm{Z}}$ are the mean vectors of $Y$ and $Z$, respectively; $\Sigma_{\mathrm{YZ}}$ is the matrix of covariance between $\boldsymbol{Y}$ and $\boldsymbol{Z}$, while $\Sigma_{\mathrm{zZ}}$ is the dispersion matrix of $\boldsymbol{Z}$. At every time point, we perform a linear regression of the log-transformed values of each of the two phenotypes (available as well as imputed) on age, smoking status, and medication indicator based only on those observations for which data were available on all the covariates and thereby obtain the residuals of each of the regressions.

\section{Test for association using binomial regression}

The phenotypes for our association analyses are the adjusted SBP and DBP levels at each time point obtained using the algorithm described in the preceding section. We propose a binomial regression framework to test for association of a SNP with a multivariate phenotype. Suppose $\boldsymbol{X}=\left(X_{1}, X_{2}, X_{3}, \ldots, X_{\mathrm{k}}\right)$ denotes a vector of $k$ phenotypes and $N$ denotes the number of copies of the minor allele $(0,1$, or 2$)$ at a SNP. We model the conditional distribution of $\boldsymbol{N}$ given $\boldsymbol{X}$ as binomial with parameters 2 and $\boldsymbol{p}(\boldsymbol{X})$ where, $\boldsymbol{p}(\boldsymbol{X})$ is a logistic link function given by:

$$
\frac{\exp \left(\beta_{0}+\sum_{i=1}^{k} \beta_{i} X_{i}\right)}{1+\exp \left(\beta_{0}+\sum_{i=1}^{k} \beta_{i} X_{i}\right)}
$$

The test for association is equivalent to testing $H_{0}$ : $\beta_{1}=\beta_{2}=\ldots=\beta_{\mathrm{k}}=0$ versus $H_{1}$ : not $H_{0}$ and the log-likelihood ratio test statistic is distributed as chi-squares with $k$ degrees of freedom under the null hypothesis. We compare the relative performances of 5 phenotype vectors in detecting association: (a) $T_{1}$ : the adjusted SBP levels at the 4 time points; (b) $T_{2}$ : the adjusted DBP levels at the 4 time points; (c) $T_{3}$ : the adjusted SBP levels summarized by the first 2 principal components across the four time points; (d) $T_{4}$ : the adjusted DBP levels summarized by the first two principal components across the four time points; and (e) $T_{5}$ : the adjusted SBP as well as the adjusted DBP levels summarized by a bivariate phenotype comprising the first 2 principal components corresponding to each of the phenotypes across the four time points. The above choice of the principal components is motivated by the fact that approximately $75 \%$ of the variation in each of the two phenotypes is explained by the corresponding first two principal components. Because the sample size is not very large, we consider only common variants for our association analyses and select only those SNPs with a minor allele frequency of at least 0.01. We also exclude those SNPs that provide significant evidence of departure from Hardy-Weinberg equilibrium (based on the Bonferroni corrected threshold at the genome-wide level). Thus, the tests for association based on the proposed binomial regression model are carried out on 426,193 SNPs. To correct for multiple testing, we use the false discovery rate (FDR) procedure [6] with an overall rate of 0.05 .

\section{Results}

Among the five phenotype vectors considered, the one comprising the first two principal components of both SBP and DBP levels $\left(T_{5}\right)$ provides the most promising association findings. Table 1 presents the five most significant association findings corresponding to each choice of phenotype vector. The only SNP that exhibits significant evidence of association at the genome-wide level is $r s 12634258$ on chromosome 3 (adjusted FDR of 0.047) with $T_{5}$. We observe that this SNP also ranks among the top five SNPs in terms of lowest unadjusted $p$-values when $T_{3}$ (the first two principal components of the SBP levels at the four time points) is analyzed.

\section{Conclusions}

We have developed a binomial regression model that can incorporate multiple phenotypes for association analysis of the multivariate phenotype vector. The method has several advantages: it neither involves any modeling of 
Table 1 Five most significant association findings using the different phenotype vectors

\begin{tabular}{|c|c|c|c|c|}
\hline$T_{1}$ & $T_{2}$ & $T_{3}$ & $T_{4}$ & $T_{5}$ \\
\hline $\begin{array}{l}\mathrm{rs} 630685 \\
\left(3 \times 10^{-6}, 1 \mathrm{q} 42.1\right)\end{array}$ & $\begin{array}{l}\text { rs7928749 } \\
\left(1 \times 10^{-6}, 11 \mathrm{q} 22.2\right)\end{array}$ & $\begin{array}{l}\text { rs11233253 } \\
\left(4.9 \times 10^{-6}, 11 \mathrm{q} 14.1\right)\end{array}$ & $\begin{array}{l}\text { rs } 4239000 \\
\left(9.3 \times 10^{-6}, 17 q 25\right)\end{array}$ & $\begin{array}{l}\text { rs12634258 } \\
\left(1 \times 10^{-7}, 3 p 14.2\right)\end{array}$ \\
\hline $\begin{array}{l}\text { rs1789956 } \\
\left(3.5 \times 10^{-6}, 21 \mathrm{q} 22.3\right)\end{array}$ & $\begin{array}{l}\text { rs } 1129174 \\
\left(5.9 \times 10^{-6}, 3 p 25\right)\end{array}$ & $\begin{array}{l}\mathrm{rs} 11212913 \\
\left(7.7 \times 10^{-6}, 11 \mathrm{q} 22.3\right)\end{array}$ & $\begin{array}{l}\text { rs } 6590606 \\
\left(2 \times 10^{-5}, 11 q 25\right)\end{array}$ & $\begin{array}{l}\text { rs12153302 } \\
\left(1.1 \times 10^{-6}, 5 q 13.2\right.\end{array}$ \\
\hline $\begin{array}{l}\text { rs } 3829088 \\
\left(3.8 \times 10^{-6}, 9 p 21\right)\end{array}$ & $\begin{array}{l}\text { rs6791435 } \\
\left(6.4 \times 10^{-6}, 3 p 25.1\right)\end{array}$ & $\begin{array}{l}\text { rs9632874 } \\
\left(9.4 \times 10^{-6}, 9 p 22.3\right)\end{array}$ & $\begin{array}{l}\text { rs7117235 } \\
\left(2.1 \times 10^{-5}, 11 \mathrm{q} 25\right)\end{array}$ & $\begin{array}{l}\text { rs } 12652232 \\
\left(3.3 \times 10^{-6}, 5 q 35.3\right.\end{array}$ \\
\hline $\begin{array}{l}\text { rs12591997 } \\
\left(4.1 \times 10^{-6}, 15 q 21.2\right)\end{array}$ & $\begin{array}{l}\text { rs } 4685160 \\
\left(9.5 \times 10^{-6}, 3 p 25.1\right)\end{array}$ & $\begin{array}{l}\text { rs16960959 } \\
\left(1.1 \times 10^{-5}, 17 p 11.2\right)\end{array}$ & $\begin{array}{l}\text { rs1870301 } \\
\left(2.9 \times 10^{-5}, 15 q 26.2\right)\end{array}$ & $\begin{array}{l}\text { rs } 7541416 \\
\left(3.9 \times 10^{-6}, 1 \mathrm{p} 36.1\right.\end{array}$ \\
\hline $\begin{array}{l}\text { rs12915086 } \\
\left(6.2 \times 10^{-6}, 15 q 26\right)\end{array}$ & $\begin{array}{l}\text { rs894177 } \\
\left(1.6 \times 10^{-5}, 3 q 24\right)\end{array}$ & $\begin{array}{l}\text { rs12634258 } \\
\left(1.2 \times 10^{-5}, 3 p 14.2\right)\end{array}$ & $\begin{array}{l}\mathrm{rs} 12153302 \\
\left(3.9 \times 10^{-5}, 5 \mathrm{q} 13.2\right)\end{array}$ & $\begin{array}{l}\text { rs } 7644778 \\
\left(4.6 \times 10^{-6}, 3 q 28\right)\end{array}$ \\
\hline
\end{tabular}

The figures in parentheses represent the unadjusted $p$-values and chromosomal locations, respectively.

the correlation structure within the components of the multivariate phenotype as required in likelihood-based approaches nor does it compromise on the biological interpretation by transforming the multivariate phenotype to principal components.

The SNP rs12634258 that exhibits the most promising evidence of association with multiple definitions of the phenotype vector is located in the intergenic region between the genes PTPRG (receptor-type tyrosine-protein phosphatase gamma) and FHIT (fragile histidine triad) on 3p14.2. The RNA expression in PTPRG has been reported to be upregulated for arrhythmogenic right ventricular cardiomyopathy in humans [7]. On the other hand, the RNA expression of FHIT is downregulated in mouse for low blood pressure [8]. Although we obtain only one association finding that satisfies the genome-wide FDR threshold of 0.05 , it is likely that the sample size is grossly inadequate to detect associations for a complex phenotype.

While constructing the phenotype vectors, we had considered the first two principal components of the blood pressure levels across the four time points. As mentioned earlier, these two principal components explain approximately $75 \%$ of the total variation of blood pressure levels across the time points. Even though the first principal component explains approximately $55 \%$ of the variation, the first 3 principal components explain approximately $90 \%$ of the variation. Although for brevity we do not present the association findings for the different choices of the principal components, we find that the SNP rs7644778 ranks among the top two association findings with the first principal component of each of SBP and DBP levels across the four time points, albeit not significant at the genomewide level. We note that this SNP also exhibits evidence of association with first two principal components of each of the two phenotypes. The SNP is located in the gene TPRG1 (tumor protein p63 regulated 1). The RNA expression of this gene has been reported to be upregulated for cardiomyopathy in humans. On the other hand, the five most significant findings with the first two principal components are identical to those with the first three principal components, but none of them is significant at the genome-wide level. This may be explained by the fact that the test with 3 principal components for each of the two phenotypes has two additional degrees of freedom under the null hypothesis and may suffer from loss in power.

Among the five phenotype vectors $\left(T_{1}, T_{2}, T_{3}, T_{4}\right.$ and $T_{5}$ ), we find that $T_{5}$ (ie, the phenotype that considers both SBP as well as DBP levels simultaneously) provides the most significant association findings (in terms of $p$-value). Even though the empirical findings seem to suggest that association analyses based on a multivariate phenotype comprising traits modulated by common genetic variants are likely to be more powerful than univariate analyses of the constituent traits of the multivariate phenotype vector, such a hypothesis can only be validated using extensive simulations. We have performed some independent simulations on 500 unrelated individuals under a few pleiotropic bivariate phenotype models: (a) the constituent traits have a bivariate normal distribution, (b) both are distributed as chi-squares, and (c) 1 trait has a normal distribution, while the other is binary. Our preliminary results based on 1000 replications indicate that our proposed method maintains the correct false-positive rates under all the phenotype models. We also find that simultaneous analyses of both the phenotypes as covariates consistently yield more power compared to analyzing each univariate trait using the binomial regression. On the other hand, the power of the proposed method based on both phenotypes is higher, similar, or marginally lower compared to that based on the first principal component, depending on whether the correlation between the traits is low (less than 0.25), medium (between 0.25 and 0.5 ) or high (greater than 0.5 ). The above phenomenon was observed irrespective of the bivariate phenotype model considered. When the gene effects are in different directions for the two phenotypes (the minor homozygous genotype corresponds to high 
values of 1 phenotype, but low values of the other phenotype), the first principal component performs very poorly and may yield lower power compared to analyzing univariate phenotypes. However, further simulations based on higher dimensional phenotype vectors need to carried out to obtain greater insights into the relative advantages of the proposed method using the multivariate phenotype vector over data reduction approaches such as using a subset of the principal components of the phenotype vector.

\section{Competing interests}

The authors declare that they have no competing interests.

\section{Authors' contributions}

SG and AM developed the proposed method. AM and IM wrote the computer codes and performed the data analyses. AM participated in the compilation and interpretation of the results. All authors read and approved the final manuscript.

\section{Acknowledgements}

This work was partially supported by the Council of Scientific and Industrial Research (CSIR) fellowship 09/093(0112)/2008-EMR-I to Arunabha Majumdar. The GAW18 whole genome sequence data were provided by the T2DGENES Consortium, which is supported by NIH grants U01 DK085524, U01 DK085584, U01 DK085501, U01 DK085526, and U01 DK085545. The other genetic and phenotypic data for GAW18 were provided by the San Antonio Family Heart Study and San Antonio Family Diabetes/Gallbladder Study, which are supported by NIH grants P01 HL045222, R01 DK047482, and R01 DK053889. The Genetic Analysis Workshop is supported by NIH grant R01 GM031575. The GAW18 whole genome sequence data were provided by the T2D-GENES Consortium which is supported by $\mathrm{NIH}$ grants U01 DK085524, U01 DK085584, U01 DK085501, U01 DK085526, and U01 DK085545. The other genetic and phenotypic data for GAW18 were provided by the San Antonio Family Heart Study and San Antonio Family Diabetes/Gallbladder Study, which are supported by NIH grants P01 HL045222, R01 DK047482, and R01 DK053889. The Genetic Analysis Workshop is supported by NIH grant R01 GM031575.

This article has been published as part of BMC Proceedings Volume 8 Supplement 1, 2014: Genetic Analysis Workshop 18. The full contents of the supplement are available online at http://www.biomedcentral.com/bmcproc/ supplements/8/S1. Publication charges for this supplement were funded by the Texas Biomedical Research Institute.

Published: 17 June 2014

\section{References}

1. Yang Q, Wu H, Guo CY, Fox CS: Analyze multivariate phenotypes in genetic association studies by combining univariate association tests. Genet Epidemiol 2010, 34:434-454.

2. Almasy L, Blangero J: Variance components methods for analysis of complex phenotypes. Cold Spring Harb Protoc 2010, 5:77.

3. Minica CC, Boosma DI, van der Sluis S, Dolan CV: Genetic association in multivariate phenotypic data: power in five models. Twin Res Hum Genet 2010, 13:525-543.

4. Elston RC, Buxbaum S, Jacobs KB, Olson JM: Haseman and Elston revisted. Genet Epidemiol 2000, 19:1-17.

5. Dempster AP, Laird NM, Rubin DB: Maximum likelihood from incomplete data via the EM Algorithm. J R Stat Soc Series B Stat Methodol 1977, 39:1-38.

6. Benjamini $Y$, Hochberg $Y$ : Controlling the false discovery rate: a practical and powerful approach to multiple test. J R Stat Soc Series B Stat Methodol 1995, 57(1):289-300.

7. Gaertner A, Schwientek P, Ellinghaus P, Summer H, Golz S, Kassner A, Schulz U, Gummert J, Milting H: Myocardial transcriptome analysis of human arrhythmogenic right ventricular cardiomyopathy. Physiol Genomics 2012, 44:99-109.
8. Puig O, Wang IM, Cheng P, Zhou P, Roy S, Cully D, Peters M, Benita Y, Thompson J, Cai TQ: Transcriptome profiling and network analysis of genetically hypertensive mice identifies potential pharmacological targets of hypertension. Physiol Genomics 2010, 42:24-32.

doi:10.1186/1753-6561-8-S1-S74

Cite this article as: Majumdar et al:: Association mapping of blood pressure levels in a longitudinal framework using binomial regression. BMC Proceedings 2014 8(Suppl 1):S74.

\section{Submit your next manuscript to BioMed Central and take full advantage of:}

- Convenient online submission

- Thorough peer review

- No space constraints or color figure charges

- Immediate publication on acceptance

- Inclusion in PubMed, CAS, Scopus and Google Scholar

- Research which is freely available for redistribution 